Polymer Journal, Vol. 7, No. 6, pp 637-640 (1975)

\title{
Asymptotic Form of the Intrinsic Viscosity of a Once-Broken Rod
}

\author{
Nobuko TAkI and Hiroshi Fujrta \\ Department of Polymer Science, Osaka University, Toyonaka, Japan.
}

(Received May 26, 1975)

\begin{abstract}
The intrinsic viscosity of a once-broken rod with equal rod sections connected by a flexible joint is calculated by means of the method developed by Yamakawa and Fujii. It is shown that the introduction of a flexible portion into the midpoint of a straight rod decreases the intrinsic viscosity by a factor of $3 / 8$ at the limit of large ratios of length to diameter, differing from the $\mathbf{Y} u-$ Stockmayer theory which predicts a reduction as small as about 0.15 . The present value conforms to Hassager's obtained recently by a different analysis.
\end{abstract}

KEY WORDS Intrinsic Viscosity / One-Broken Rod / YamakawaFujii's Method /

According to theoretical calculation by $\mathrm{Yu}$ and Stockmayer, ${ }^{1}$ the intrinsic viscosity of a macromolecule composed of two rod sections of equal length connected by a flexible joint, at the limit of infinite length, approaches a value about $15 \%$ smaller than that of a straight rod of the same length and dimeter. This reduction in intrinsic viscosity is rather unexpectedly small if one considers that there occurs a $37.5 \%$ decrease in the mean-square radius gyration, $\left\langle S^{2}\right\rangle$, when a flexible joint is introduced into the midpoint of a straight rod. The present paper aims at examining the validity of $\mathrm{Yu}-$ Stockmayer's conclusion by a different approach. Here the intrinsic viscosity is calculated from an asymptotic solution to the integral equation derived recently by Yamakawa and $\mathrm{Fujii}^{2}$ using OseenBurgers approximation to polymer hydrodynamics. ${ }^{3,4}$

After completion of this work, we have become aware of the fact that Hassager ${ }^{5}$ had arrived at a conclusion similar to that given in the present paper. However, since our approach is quite different from his and, moreover, since our calculation takes account of the hydrodynamic interactions between different rod sections that he ignored, it is hoped that the present article is worth publication as a meaningful addition to the literature on polymer solution theory.

\section{BASIC EQUATIONS}

Consider a molecular model composed of two smooth rods of equal length $L / 2$ and diameter $d$ connected by a flexible joint. According to Yamakawa and Fujii, ${ }^{2}$ the intrinsic viscosity, $[\eta]_{b}$, of this once-broken rod can be calculated from

$$
[\eta]_{\mathrm{b}}=\frac{\pi N_{\mathrm{A}}}{M} \int_{-L / 2}^{L / 2} \phi(s, s) \mathrm{d} s
$$

Here, $N_{\mathrm{A}}$ is Avogadro's number, $M$ is the molecular weight, and $\phi(s, s)$ is a function of $s$ which can be determined from YamakawaFujii's integral equation

$$
\int_{-L / 2}^{L / 2} K(s, t) \phi\left(t, s^{\prime}\right) \mathrm{d} t=\left\langle\boldsymbol{S}(s) \cdot \boldsymbol{S}\left(s^{\prime}\right)\right\rangle
$$

with

$$
K(s, t)=\left\langle|\boldsymbol{R}(s, t)-\boldsymbol{r}(s)|^{-1}\right\rangle
$$

In these equations, $\boldsymbol{R}(s, t)$ is the vector drawn from a contour point $s$ to a contour point $t, r(s)$ is the normal radius vector at the point $s, S(s)$ is the vector from the center of mass of the rod to the point $s$ and the angular bracket designates the average over all possible configurations of the rod. The origin for contour lengths $s, s^{\prime}$ and $t$ is chosen at the joint of the two rods, with the ends of the entire rod being given the $t$ values of $+L / 2$ and $-L / 2$. We write down eq 2 for the two cases in which the contour points $s$ and $s^{\prime}$ are on the same rod section I and on the different rod sections I and II. Then, we have 


$$
\begin{aligned}
& \int_{0}^{L / 2}\left[K(s, t) \phi\left(t, s^{\prime}\right)+K(s,-t) \phi\left(-t, s^{\prime}\right)\right] \mathrm{d} t \\
& \quad=\left\langle\boldsymbol{S}(s) \cdot \boldsymbol{S}\left(s^{\prime}\right)\right\rangle
\end{aligned}
$$

if $s$ and $s^{\prime}$ are on the rod section $\mathrm{I}$, and

$$
\begin{aligned}
& \int_{0}^{L / 2}\left[K(s, t) \phi\left(t,-\left|s^{\prime}\right|\right)+K(s,-t) \phi\left(-t,-\left|s^{\prime}\right|\right)\right] \mathrm{d} t \\
& \quad=\left\langle\boldsymbol{S}(s) \cdot \boldsymbol{S}\left(-\left|s^{\prime}\right|\right)\right\rangle
\end{aligned}
$$

if $s$ is on the rod section I and $s^{\prime}\left(=-\left|s^{\prime}\right|\right)$ is on the rod section II. From the definition of $\phi$ we find that

$$
\phi\left(t, s^{\prime}\right)=\phi\left(-t,-s^{\prime}\right) \quad \phi\left(t,-s^{\prime}\right)=\phi\left(-t, s^{\prime}\right)
$$

provided that $t$ and $s^{\prime}$ are positive. Hence it follows from eq 4 and 5 that

$$
\begin{aligned}
& \int_{0}^{L / 2} J_{1}(s, t) \xi_{1}\left(t, s^{\prime}\right) \mathrm{d} t=X_{1}\left(s, s^{\prime}\right) \\
& \int_{0}^{L / 2} J_{2}(s, t) \xi_{2}\left(t, s^{\prime}\right) \mathrm{d} t=X_{2}\left(s, s^{\prime}\right)
\end{aligned}
$$

where

$$
\begin{aligned}
J_{1}(s, t) & =K(s, t)+K(s,-t) \\
J_{2}(s, t) & =K(s, t)-K(s,-t) \\
\xi_{1}\left(t, s^{\prime}\right) & =\phi\left(t, s^{\prime}\right)+\phi\left(t,-s^{\prime}\right) \\
\xi_{2}\left(t, s^{\prime}\right) & =\phi\left(t, s^{\prime}\right)-\phi\left(t,-s^{\prime}\right)
\end{aligned}
$$

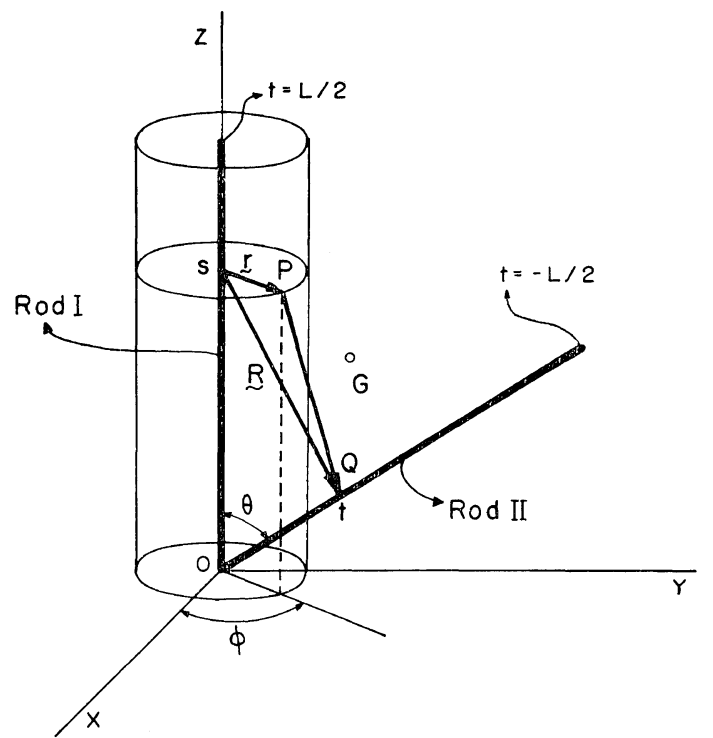

Figure 1. The polar coordinate system used for the computation of $K(s, t)$ when $s$ is positive and $t$ is negative. The point $G$ denotes the center of mass for the entire rod.

$$
\begin{aligned}
& X_{1}\left(s, s^{\prime}\right)=\left\langle\boldsymbol{S}(s) \cdot \boldsymbol{S}\left(s^{\prime}\right)\right\rangle+\left\langle\boldsymbol{S}(s) \cdot \boldsymbol{S}\left(-s^{\prime}\right)\right\rangle \\
& X_{2}\left(s, s^{\prime}\right)=\left\langle\boldsymbol{S}(s) \cdot \boldsymbol{S}\left(s^{\prime}\right)\right\rangle-\left\langle\boldsymbol{S}(s) \cdot \boldsymbol{S}\left(-s^{\prime}\right)\right\rangle
\end{aligned}
$$

with $t, s$ and $s^{\prime}$ being understood to be positive. Equations 7 and 8 are the separate integral equations for $\xi_{1}\left(t, s^{\prime}\right)$ and $\xi_{2}\left(t, s^{\prime}\right)$, and their solution allows determination of $\phi\left(t, s^{\prime}\right)$ and $\phi\left(t,-s^{\prime}\right)$ from eq 10 , which in turn may be used to compare $[\eta]_{\mathrm{b}}$ from eq 1 .

Now, $K(s, t)(s, t>0)$ is given by

$$
K(s, t)=\frac{1}{\left[(s-t)^{2}+r^{2}\right]^{1 / 2}}
$$

To obtain $K(s,-t)(s, t>0)$ it is convenient to use the polar coordinate system shown in Figure 1. The rod section I lies on the $z$-axis with the joint at the origin 0 , and the rod section II points in an arbitary direction having polar angle $\theta$ and azimuthal angle $\phi$. Then, we can write $K(s,-t)$ in the form

$K(s,-t)$

$=\frac{1}{4 \pi} \int_{0}^{\pi} \int_{0}^{2 \pi} \frac{\sin \theta \mathrm{d} \theta \mathrm{d} \phi}{\left[r^{2}+t^{2}+s^{2}-2 s t \cos \theta-2 r t \sin \theta \sin \phi\right]^{1 / 2}}$

After some complex manipulations, eq 13 yields $K(s,-t)$

$$
=\frac{s}{2 t\left(s^{2}+r^{2}\right)}\left\{\left[(s+t)^{2}+r^{2}\right]^{1 / 2}-\left[(s-t)^{2}+r^{2}\right]^{1 / 2}\right\}
$$

with the neglect of terms which may be left unevaluated for the final purpose of the present calculation.

Both $\left\langle\boldsymbol{S}(s) \cdot \boldsymbol{S}\left(s^{\prime}\right)\right\rangle$ and $\left\langle\boldsymbol{S}(s) \cdot \boldsymbol{S}\left(-s^{\prime}\right)\right\rangle$ for $s>0$ and $s^{\prime}>0$ are readily obtained, giving

$$
\begin{gathered}
\left\langle\boldsymbol{S}(s) \cdot \boldsymbol{S}\left(s^{\prime}\right)\right\rangle=(1 / 32)\left[L^{2}-4 L\left(s+s^{\prime}\right)\right]+s s^{\prime} \\
\left\langle\boldsymbol{S}(s) \cdot \boldsymbol{S}\left(-s^{\prime}\right)\right\rangle=(1 / 32)\left[L^{2}-4 L\left(s+s^{\prime}\right)\right]
\end{gathered}
$$

With these results and the changes of variables as

$$
\begin{aligned}
& x=(4 s / L)-1 \\
& y=\left(4 s^{\prime} / L\right)-1 \\
& z=(4 t / L)-1
\end{aligned}
$$

eq 7 and 8 yield

$$
\begin{aligned}
& \int_{-1}^{1}\left[R_{1}(x, z)+R_{2}(x, z)\right] \psi_{1} \mathrm{~d} z=x \\
& \int_{-1}^{1}\left[R_{1}(x, z)-R_{2}(x, z)\right] \psi_{2} \mathrm{~d} z=x+1
\end{aligned}
$$




\section{Once-Broken Rod}

where

$$
\begin{aligned}
& \psi_{1}(z)=\left(16 / L^{2} y\right) \xi_{1}(s, t) \\
& \phi_{2}(z)=\left[16 / L^{2}(y+1)\right] \xi_{2}(s, t)
\end{aligned}
$$

and

$$
\begin{aligned}
& R_{1}(x, z)=\left[(x-z)^{2}+4(d / L)^{2}\right]^{-1 / 2} \\
& R_{2}(x, z)=(x+1)\left\{2(z+1)\left[(x+1)^{2}+4(d / L)^{2}\right]\right\}^{-1} \\
& \times\left\{\left[(x+z+2)^{2}+4(d / L)^{2}\right]^{1 / 2}\right. \\
&\left.-\left[(x-z)^{2}+4(d / L)^{2}\right]^{1 / 2}\right\}
\end{aligned}
$$

It is a simple matter to show that eq 1 can be rewritten in terms of $\phi_{1}(z)$ and $\phi_{2}(z)$ as follows:

$$
[\eta]_{\mathrm{b}}=\frac{\pi N_{\mathrm{A}} L^{3}}{64 M} \int_{-1}^{1}\left[x \psi_{1}(x)+(x+1) \psi_{2}(x)\right] \mathrm{d} x
$$

Thus the task is to solve the integral equations 18 and 19 for $\psi_{1}(z)$ and $\psi_{2}(z)$.

\section{ASYMPTOTIC SOLUTIONS}

For this purpose we expand these unknown functions $\psi_{n}$ in terms of Legendre polynominals $P_{i}(z)$, i.e.,

$$
\psi_{n}(z)=\sum_{i=0}^{\infty} b_{n i} P_{i}(z) \quad(n=1,2)
$$

and apply the same procedure as described by Yamakawa and $\mathrm{Fujii}^{2}$ and also by $\mathrm{Taki}^{6}$ for straight rods. Then, eq 18 and 19 allow the coefficients $b_{n i}$ to be expressed in terms of $a_{n, i j}$, where

$a_{n, i j}=\int_{-1}^{1} \int_{-1}^{1}\left[R_{1}(x, z) \pm R_{2}(x, z)\right] P_{i}(x) P_{j}(z) \mathrm{d} x \mathrm{~d} z$

with the plus sign in the brackets for $n=1$ and the minus sign for $n=2$.

On the other hand, substitution of eq 24 into eq 23 and use of the relations between $b_{n i}$ and $a_{n, i j}$ so obtained leads to

$$
\begin{aligned}
{[\eta]_{\mathrm{b}}=} & \frac{\pi N_{\mathrm{A}} L^{3}}{144 M}\left[A_{1}{ }^{11} /\left|A_{1}\right|\right. \\
& \left.+\left(9 A_{2}{ }^{00}+3 A_{2}{ }^{01}+3 A_{2}{ }^{10}+A_{2}{ }^{11}\right) /\left|A_{2}\right|\right]
\end{aligned}
$$

where $\left|A_{n}\right|$ is the determinant whose elements are $a_{n, i j}(i, j=0,1, \ldots)$ and $A_{n}^{i j}$ is the cofactor for the element $a_{n}, i j$.

For the derivation of an asymptotic form of $[\eta]_{\mathrm{b}}$ valid for large $L / d$, both $\left|A_{1}\right|$ and $\left|A_{2}\right|$ may be replaced by $2 \times 2$ determinants, and $a_{n, i j}(i, j=$
$0,1)$ with dropping the terms multiplied by $d / L$ are written as follows:

$$
\begin{aligned}
& a_{1,00}=4[\ln (L / d)+\ln 2] \\
& a_{1,01}=a_{1,10}=-1 \\
& a_{1,11}=(4 / 3)[\ln (L / d)+\ln 2-11 / 6] \\
& a_{2,00}=4[\ln (L / d)+\ln 2-2] \\
& a_{2,01}=a_{2,10}=1 \\
& a_{2,11}=(4 / 3)[\ln (L / d)+\ln 2-17 / 6]
\end{aligned}
$$

Then we obtain from eq 26

$$
[\eta]_{\mathrm{b}}=\frac{5 \pi N_{\mathrm{A}} L^{3}}{192 M} \frac{1}{\ln (L / d)+\ln 2-11 / 6}
$$

which is the final result of the present calculation, valid asymptotically for a once broken rod with equal and long rod sections connected by a completely flexible joint. The constant $\ln 2-11 / 6$ next to the term $\ln (L / d)$ in eq 28 actually represents the leading correction for hydrodynamic interactions between the two rod sections. Yu and Stockmayer ${ }^{1}$ have not extended their calculation to such a correction term. More important is the fact that, when divided by the Yamakawa-Fujii expression for $[\eta]_{s},{ }^{1}$ the intrinsic viscosity of a straight rod of length $L$ and diameter $d$, eq 28 tends to $5 / 8$ at the limit of infinite $L / d$, differing appreciably from the value (about 0.85 ) reported by $\mathrm{Yu}$ and Stockmayer. It is to be remarked, however, that the present calculation is based on the starting equations, eq 2 with eq 3 , which were derived by preaveraging the Oseen tensor for hydrodynamic interactions. If use can be made of an equation not involving the preaveraged Oseen tensor, the constants $5 / 192$ and $\ln 2-11 / 6$ obtained above will be subject to certain changes.

As noted before, Hassager ${ }^{5}$ has recently arrived at the same value $5 / 8$ for the asymptotic ratio of $[\eta]_{\mathrm{b}}$ to $[\eta]_{\mathrm{s}}$ using a different method of analysis. He describes why $\mathrm{Yu}$ and Stockmayer were led to an incorrect result. However it may be added that Hassager's formalism failed to predict the important $\ln (L / d)$ dependence of both $[\eta]_{\mathrm{s}}$ and $[\eta]_{\mathrm{b}}$, evident in Yamakawa-Fujii's expression for the former and ours for the latter.

We wish to thank Professor H. Yamakawa, Department of Polymer Chemistry, Kyoto University, for calling attention to Hassager's paper. 
N. TAKI and H. FUJITA

\section{REFERENCES}

1. H. Yamakawa and M. Fujii, Macromolecules, 7, 128 (1974).

2. H. Yu and W. H. Stockmayer, J. Chem. Phys., 47, 1369 (1967).
3. H. Fujita, J. Polym. Sci., Part A-2, 11, 899 (1973).

4. H. Yamakawa and M. Fujii, Macromolecules, 6, 407 (1973).

5. O. Hassager, J. Chem. Phys., 60, 2111 (1974).

6. N. Taki, Polymer J., 7, 586 (1975). 